\title{
SPL Shape Terminology
}

National Cancer Institute

\section{Source}

National Cancer Institute. SPL Shape Terminology. NCI Thesaurus. Code C54454.

Terminology used for representation of the information on pharmaceutical product shape in the framework of the Structured Product Labeling documents. 